Rajneesh Kakar*

\title{
Analysis of the effect of gravity and nonhomogeneity on Rayleigh waves in higher-order elastic-viscoelastic half-space
}

\begin{abstract}
In this study, the coupled effects of gravity and nonhomogeneity on Rayleigh waves in an anisotropic layer placed over an isotropic viscoelastic half-space of higher order are discussed. The dispersion properties of waves are derived. The numerical solutions are also discussed for the Rayleigh waves. It is observed that the phase velocity of Rayleigh waves is influenced quite remarkably by the presence of inhomogeneity, gravity, and strain rates of the strain parameters in the half-space.
\end{abstract}

Keywords: anisotropy; elasticity; inhomogeneity; Rayleigh waves; viscoelasticity.

DOI 10.1515/jmbm-2014-0010

\section{Introduction}

The theory of elasticity and viscoelasticity is useful in the field of solid mechanics. Any disturbance in the earth interior may be a cause for seismic wave propagation. The propagation of these waves is not only influenced by the anisotropy of the medium but also by the intrinsic viscosity of the medium [1]. Thus, in order to describe seismic wave propagation more accurately, it is necessary to consider both anisotropic characteristics and viscoelastic properties. Many years ago, Bromwich [2] considered, in particular, the effect of gravity on wave propagation and treating the gravity as a type of body force in an elastic solid medium. Love [3] extended the work of Bromwich and showed that the Rayleigh wave velocity is affected by the gravity field. Biot [4] discussed the Rayleigh waves under gravity and the initial hydrostatic stress. Das and Sengupta [5] have discussed the Rayleigh, Love, and Stoneley types of waves propagating in general viscoelastic media of higher order. Dutta [6] have studied the propagation of Love waves in a nonhomogeneous internal stratum of finite depth lying between two

*Corresponding author: Rajneesh Kakar, Department of Physics, GNA University, Phagwara, 163, Phase-1, Chotti Baradari, Garah Road, Jalandhar-144022, India, e-mail: rajneesh.kakar@gmail.com semi-infinite isotropic media, assuming that the rigidity and density are varying exponentially with depth. Kakar et al. [7] have shown the effect of gravity on the surface wave in fiberreinforced viscoelastic media of the nth order. Kakar and Kakar [8] have discussed the effects of nonhomogeneity, viscosity, and gravity as well as the magnetic and thermal fields on the Stoneley, Rayleigh, and Love waves. Vishwakarma and Gupta [9] have investigated the effect of the rigid boundary on Rayleigh waves. Gupta [10] discussed the propagation of Rayleigh waves in an initial stressed layer over an initial stressed half-space. Sofiyev et al. [11] considered the nonlinear buckling behavior of laminated orthotropic conical shells in the presence of gravity.

In this work, an attempt is made to study the behavior of Rayleigh waves propagating in an inhomogeneous anisotropic elastic layer lying over an isotropic viscoelastic solid medium of the nth order under gravity when the upper boundary plane is considered as a free surface. In the layer, it is assumed that the elastic coefficients and the density vary exponentially. The dispersion relation is obtained in a determinant form. The graphs are plotted between the phase velocity $c$ and the wave number $k$ for different values of inhomogeneity parameters for a particular model, and the effects of inhomogeneity and gravity are studied. The effects of inhomogeneity and depth on the phase velocity are also shown in the corresponding figures.

\section{Formulation of the problem}

We consider an inhomogeneous anisotropic elastic layer of the finite thickness $h$ lying over an isotropic half-space of a viscoelastic material. The interface of these two media is considered to be at $z=0$, whereas the free surface is at $z=-h$. The $z$-axis is directed vertically downward and the $x$-axis is assumed to be in the direction of the propagation of the wave with velocity $c$ (as shown in Figure 1). For Rayleigh waves, the displacement does not depend on $y$, and if $(u, v$, $w)$ denotes the displacement components at any point $P(x$, $y, z)$ of the medium, then $v=0$ and $u, w$ are functions of $(x$, $z$ ) and $t$ only. 


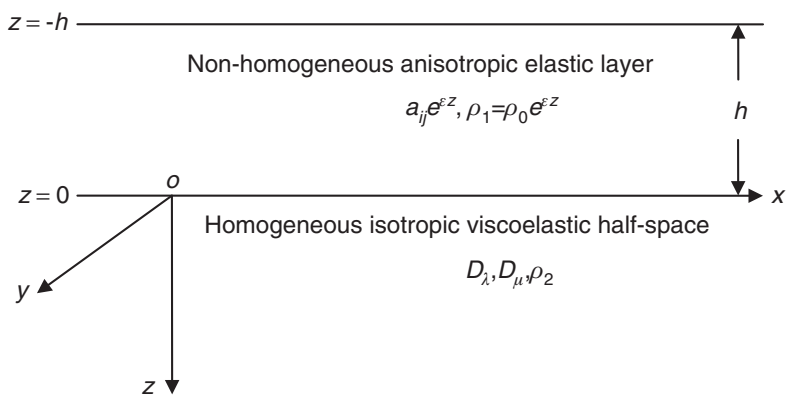

Figure 1 Geometry of the problem.

\section{Solution of the problem}

\subsection{Solution for the layer}

It is assumed that the gravitational field introduces a hydrostatic initial stress, which is produced by a slow creep process where the shearing stresses tend to become small or vanish after a long period of time. The equilibrium conditions for the initial stress are

$$
\begin{gathered}
\frac{\partial \tau}{\partial x}=0, \frac{\partial \tau}{\partial z}+\rho g=0, \\
\tau_{x x}=\tau_{z z}=\tau, \tau_{x z}=0 .
\end{gathered}
$$

The dynamical equations of motion governing the propagation of three-dimensional waves under the effect of gravity are given by Biot [4]:

$$
\begin{gathered}
\frac{\partial \tau_{x x}}{\partial x}+\frac{\partial \tau_{x y}}{\partial y}+\frac{\partial \tau_{x z}}{\partial z}+\rho_{1} g \frac{\partial w_{1}}{\partial x}=\rho_{1} \frac{\partial^{2} u_{1}}{\partial t^{2}}, \\
\frac{\partial \tau_{x y}}{\partial x}+\frac{\partial \tau_{y y}}{\partial y}+\frac{\partial \tau_{y z}}{\partial z}+\rho_{1} g \frac{\partial w_{1}}{\partial y}=\rho_{1} \frac{\partial^{2} v_{1}}{\partial t^{2}}, \\
\frac{\partial \tau_{x z}}{\partial x}+\frac{\partial \tau_{y z}}{\partial y}+\frac{\partial \tau_{z z}}{\partial z}-\rho_{1} g\left(\frac{\partial u_{1}}{\partial x}+\frac{\partial v_{1}}{\partial y}\right)=\rho_{1} \frac{\partial^{2} w_{1}}{\partial t^{2}},
\end{gathered}
$$

where $\rho_{1}$ is the density of the layer.

The stress-strain relations for an inhomogeneous anisotropic layer are of the form:

$$
\begin{aligned}
& \tau_{x x}=e^{\varepsilon z}\left\{a_{11} e_{x x}+a_{12} e_{y y}+a_{13} e_{z z}+a_{14} e_{y z}+a_{15} e_{x z}+a_{16} e_{x y}\right\}, \\
& \tau_{y y}=e^{\varepsilon z}\left\{a_{12} e_{x x}+a_{22} e_{y y}+a_{23} e_{z z}+a_{24} e_{y z}+a_{25} e_{x z}+a_{26} e_{x y}\right\}, \\
& \tau_{z z}=e^{\varepsilon z}\left\{a_{13} e_{x x}+a_{23} e_{y y}+a_{33} e_{z z}+a_{34} e_{y z}+a_{35} e_{x z}+a_{36} e_{x y}\right\}, \\
& \tau_{y z}=e^{\varepsilon z}\left\{a_{14} e_{x x}+a_{24} e_{y y}+a_{34} e_{z z}+a_{44} e_{y z}+a_{45} e_{x z}+a_{46} e_{x y}\right\},
\end{aligned}
$$

$$
\begin{aligned}
& \tau_{x z}=e^{\varepsilon z}\left\{a_{15} e_{x x}+a_{25} e_{y y}+a_{35} e_{z z}+a_{45} e_{y z}+a_{55} e_{x z}+a_{56} e_{x y}\right\}, \\
& \tau_{x y}=e^{\varepsilon z}\left\{a_{16} e_{x x}+a_{26} e_{y y}+a_{36} e_{z z}+a_{46} e_{y z}+a_{56} e_{x z}+a_{66} e_{x y}\right\},
\end{aligned}
$$

and the density is taken as $\rho_{1}=\rho_{0} e^{\varepsilon z}$.

Now, the equations of motion for the propagation of Rayleigh waves in an inhomogeneous anisotropic medium obeying Equations (2) to (5) become

$$
\begin{aligned}
a_{11} \frac{\partial^{2} u_{1}}{\partial x^{2}} & +a_{15} \frac{\partial^{2} w_{1}}{\partial x^{2}}+a_{55} \frac{\partial^{2} u_{1}}{\partial z^{2}}+a_{35} \frac{\partial^{2} w_{1}}{\partial z^{2}}+2 a_{15} \frac{\partial^{2} u_{1}}{\partial x \partial z} \\
& +\left(a_{13}+a_{55}\right) \frac{\partial^{2} w_{1}}{\partial x \partial z}+\varepsilon C_{15} \frac{\partial u_{1}}{\partial x}+\varepsilon a_{55} \frac{\partial w_{1}}{\partial x}+\varepsilon a_{55} \frac{\partial u_{1}}{\partial z} \\
& +\varepsilon a_{35} \frac{\partial w_{1}}{\partial z}+\rho_{0} g \frac{\partial w_{1}}{\partial x}=\rho_{0} \frac{\partial^{2} u_{1}}{\partial t^{2}},
\end{aligned}
$$

$$
\begin{aligned}
a_{15} \frac{\partial^{2} u_{1}}{\partial x^{2}} & +a_{55} \frac{\partial^{2} w_{1}}{\partial x^{2}}+a_{35} \frac{\partial^{2} u_{1}}{\partial z^{2}}+a_{33} \frac{\partial^{2} w_{1}}{\partial z^{2}} \\
& +\left(a_{13}+a_{55}\right) \frac{\partial^{2} u_{1}}{\partial x \partial z}+2 a_{35} \frac{\partial^{2} w_{1}}{\partial x \partial z}+\varepsilon a_{13} \frac{\partial u_{1}}{\partial x}+\varepsilon a_{35} \frac{\partial w_{1}}{\partial x} \\
& +\varepsilon a_{35} \frac{\partial u_{1}}{\partial z}+\varepsilon a_{33} \frac{\partial w_{1}}{\partial z}-\rho_{0} g \frac{\partial u_{1}}{\partial x}=\rho_{0} \frac{\partial^{2} w_{1}}{\partial t^{2}} .
\end{aligned}
$$

On seeking solution for the above equations in the form $u_{1}(x, z, t)=A(z) e^{i k(x-c t)}$ and $w_{1}(x, z, t)=B(z) e^{i k(x-c t)}$, we have

$$
\begin{aligned}
{\left[a_{55} D^{2}\right.} & \left.+\left(2 i k a_{15}+\varepsilon a_{55}\right) D+\left(\rho_{0} k^{2} c^{2}-a_{11} k^{2}+i k \varepsilon a_{15}\right)\right] A \\
& +\left[a_{35} D^{2}+\left\{i k\left(a_{13}+a_{55}\right)+\varepsilon a_{35}\right\} D\right. \\
& \left.+\left(-a_{15} k^{2}+i k \varepsilon a_{55}+i k \rho_{0} g\right)\right] B=0,
\end{aligned}
$$

$$
\begin{aligned}
{\left[a_{35} D^{2}\right.} & \left.+\left\{i k\left(a_{13}+a_{55}\right)+\varepsilon a_{35}\right\} D+\left(-a_{15} k^{2}+i k \varepsilon a_{13}-i k \rho_{0} g\right)\right] A \\
& +\left[a_{33} D^{2}+\left(2 i k a_{35}+\varepsilon a_{33}\right) D\right. \\
& \left.+\left(\rho_{0} k^{2} c^{2}-a_{55} k^{2}+i k \varepsilon a_{35}\right)\right] B=0 .
\end{aligned}
$$

Following the standard method for solving the simultaneous linear algebraic equations with constant coefficients, we write $A(z)=\phi e^{-k \delta z}, B(z)=\psi e^{-k \delta z}$, and by using Equations (8) and (9), we have

$$
\begin{aligned}
& {\left[a_{55} \delta^{2}-\left(2 i a_{15}+\frac{\varepsilon a_{55}}{k}\right) \delta+\left(\rho_{0} c^{2}-a_{11}+\frac{i \varepsilon a_{15}}{k}\right)\right] \phi} \\
& +\left[a_{35} \delta^{2}-\left\{i\left(a_{13}+C_{55}\right)+\frac{\varepsilon a_{35}}{k}\right\} \delta+\left(-a_{15}+\frac{i \varepsilon a_{55}}{k}+\frac{i \rho_{0} g}{k}\right)\right] \psi=0,
\end{aligned}
$$

$$
\begin{aligned}
& {\left[a_{35} \delta^{2}-\left\{i\left(a_{13}+a_{55}\right)+\frac{\varepsilon a_{35}}{k}\right\} \delta+\left(-a_{15}+\frac{i \varepsilon a_{33}}{k}-\frac{i \rho_{0} g}{k}\right)\right] \phi} \\
& +\left[a_{33} \delta^{2}-\left(2 i a_{35}+\frac{\varepsilon a_{33}}{k}\right) \delta+\left(\rho_{0} c^{2}-a_{55}+\frac{i \varepsilon a_{35}}{k}\right)\right] \psi=0 .
\end{aligned}
$$


In order to obtain a nontrivial solution of Equations (10) and (11), the following condition should be fulfilled:

$$
\alpha_{0} \delta^{4}+\alpha_{1} \delta^{3}+\alpha_{2} \delta^{2}+\alpha_{3} \delta+\alpha_{4}=0,
$$

where $\alpha_{0}, \alpha_{1}, \alpha_{2}, \alpha_{3}$, and $\alpha_{4}$ are given in the Appendix.

If by $\delta_{j}=(j=1, \ldots, 4)$ we denote the roots of Equation (12), the ratio of the displacement components $\frac{A_{j}}{B_{j}}$ from Equation (10) corresponding to $\delta=\delta_{i}$ is

$\frac{B_{j}}{A_{j}}=\frac{\psi_{j}}{\phi_{j}}=\frac{-\left[a_{55} \delta_{j}^{2}-\left(2 i a_{15}+\frac{\varepsilon a_{55}}{k}\right) \delta_{j}+\left(\rho_{0} c^{2}-a_{11}+\frac{i \varepsilon C_{15}}{k}\right)\right]}{\left[a_{35} \delta_{j}^{2}-\left\{i\left(a_{13}+a_{55}\right)+\frac{\varepsilon a_{35}}{k}\right\} \delta_{j}+\left(-a_{15}+\frac{i \varepsilon a_{55}}{k}+\frac{i \rho_{0} g}{k}\right)\right]} \equiv p_{j}$.

Thus, the solution of Equations (6) and (7) can be written as

$$
\begin{gathered}
u_{1}=\left(\phi_{1} e^{-k \delta_{1} z}+\phi_{2} e^{-k \delta_{2} z}+\phi_{3} e^{-k \delta_{3} z}+\phi_{4} e^{-k \delta_{4} z}\right) e^{i k(x-c t)}, \\
w_{1}=\left(p_{1} \phi_{1} e^{-k \delta_{1} z}+p_{2} \phi_{2} e^{-k \delta_{2} z}+p_{3} \phi_{3} e^{-k \delta_{3} z}+p_{4} \phi_{4} e^{-k \delta_{4} z}\right) e^{i k(x-c t)} .
\end{gathered}
$$

\subsection{Solution for the half-space}

The dynamical equations of motion for the propagation of Rayleigh waves under the effect of gravity are given by Biot [4]:

$$
\begin{gathered}
\frac{\partial \tau_{x x}}{\partial x}+\frac{\partial \tau_{x z}}{\partial z}+\rho_{2} g \frac{\partial w_{2}}{\partial x}=\rho_{2} \frac{\partial^{2} u_{2}}{\partial t^{2}}, \\
\frac{\partial \tau_{x z}}{\partial x}+\frac{\partial \tau_{z z}}{\partial z}-\rho_{2} g \frac{\partial u_{2}}{\partial x}=\rho_{2} \frac{\partial^{2} w_{2}}{\partial t^{2}}
\end{gathered}
$$

where $\rho_{2}$ is the density of the viscoelastic medium. The stress-strain relations for a general isotropic viscoelastic medium are given by the relations:

$$
\begin{gathered}
\tau_{x x}=\left(D_{\lambda}+2 D_{\mu}\right) \frac{\partial u_{2}}{\partial x}+D_{\mu} \frac{\partial w_{2}}{\partial z}, \\
\tau_{z z}=D_{\lambda} \frac{\partial u_{2}}{\partial x}+\left(D_{\lambda}+2 D_{\mu}\right) \frac{\partial w_{2}}{\partial z}, \\
\tau_{x z}=D_{\mu}\left(\frac{\partial w_{2}}{\partial x}+\frac{\partial u_{2}}{\partial z}\right),
\end{gathered}
$$

where $D_{\lambda}=\sum_{j=0}^{n} \lambda_{j} \frac{\partial^{j}}{\partial t^{j}}$ and $D_{\mu}=\sum_{j=0}^{n} \mu_{j} \frac{\partial^{j}}{\partial t^{j}}$.
The substitution of Equation (18) in Equations (16) and (17) gives

$$
\left(D_{\lambda}+2 D_{\mu}\right) \frac{\partial^{2} u_{2}}{\partial x^{2}}+\left(D_{\lambda}+D_{\mu}\right) \frac{\partial^{2} w_{2}}{\partial x \partial z}+D_{\mu} \frac{\partial^{2} u_{2}}{\partial z^{2}}+\rho_{2} g \frac{\partial w_{2}}{\partial x}=\rho_{2} \frac{\partial^{2} u_{2}}{\partial t^{2}},
$$

$D_{\mu} \frac{\partial^{2} w_{2}}{\partial x^{2}}+\left(D_{\lambda}+D_{\mu}\right) \frac{\partial^{2} u_{2}}{\partial x \partial z}+\left(D_{\lambda}+2 D_{\mu}\right) \frac{\partial^{2} w_{2}}{\partial z^{2}}-\rho_{2} g \frac{\partial u_{2}}{\partial x}=\rho_{2} \frac{\partial^{2} w_{2}}{\partial t^{2}}$.

Assuming that the solution of above equations is of the form $u_{2}(x, z, t)=F e^{i k(x-c t)}$ and $w_{2}(x, z, t)=G(z) e^{i k(x-c t)}$ and substituting in Equations (19) and (20), we have

$$
\begin{aligned}
\left\{D_{\mu}^{\prime} D^{2}+\right. & \left.\left(\rho_{2} k^{2} c^{2}-k^{2}\left(D_{\lambda}^{\prime}+2 D_{\mu}^{\prime}\right)\right)\right\} F+\left\{i k\left(D_{\lambda}^{\prime}+D_{\mu}^{\prime}\right) D\right. \\
& \left.+i k \rho_{2} g\right\} G=0, \\
\{ & \left.i k\left(D_{\lambda}^{\prime}+D_{\mu}^{\prime}\right) D-i k \rho_{2} g\right\} F+\left\{\left(D_{\lambda}^{\prime}+2 D_{\mu}^{\prime}\right) D^{2}\right. \\
& \left.+\left(\rho_{2} k^{2} c^{2}-k^{2} D_{\mu}^{\prime}\right)\right\} G=0,
\end{aligned}
$$

where $D_{\lambda}^{\prime}=\sum_{j=0}^{n} \lambda_{j}(-i k c)^{j}$ and $D_{\mu}^{\prime}=\sum_{j=0}^{n} \mu_{j}(-i k c)^{j}$.

Proceeding as above for the solution in the layer, we write $F(z)=\xi e^{-k \eta z}, G(z)=\zeta e^{-k \eta z}$ and by using Equations (21) and (22), we have

$$
\begin{gathered}
\left\{D_{\mu}^{\prime} \eta^{2}+\left(\rho_{2} c^{2}-\left(D_{\lambda}^{\prime}+2 D_{\mu}^{\prime}\right)\right)\right\} \xi+\left\{-i\left(D_{\lambda}^{\prime}+D_{\mu}^{\prime}\right) \eta+\frac{i \rho_{2} g}{k}\right\} s=0,(23) \\
-\left\{i\left(D_{\lambda}^{\prime}+D_{\mu}^{\prime}\right) \eta+\frac{i \rho_{2} g}{k}\right\} \xi+\left\{\left(D_{\lambda}^{\prime}+2 D_{\mu}^{\prime}\right) \eta^{2}+\left(\rho_{2} c^{2}-D_{\mu}^{\prime}\right)\right\} s=0,
\end{gathered}
$$

In order to obtain the nontrivial solution of Equations (23) and (24), we must solve the following biquadratic algebraic equation in $\eta$ :

$$
\beta_{0} \eta^{4}+\beta_{1} \eta^{2}+\beta_{2}=0
$$

where $\beta_{0}, \beta_{1}$, and $\beta_{2}$ are defined in the Appendix. Let $\pm \eta_{1}, \pm \eta_{2}$ be the roots of Equation (25), then

$$
\eta_{1}^{2}=\frac{-\beta_{1}-\sqrt{\beta_{1}^{2}-4 \beta_{0} \beta_{2}}}{2 \beta_{0}} \text { and } \eta_{2}^{2}=\frac{-\beta_{1}+\sqrt{\beta_{1}^{2}-4 \beta_{0} \beta_{2}}}{2 \beta_{0}} \text {. }
$$

In view of Equation (23), the ratio of the displacement components $\frac{G_{j}}{F_{j}}$, the corresponding to $\eta=\eta_{j}$ is

$$
\frac{G_{j}}{F_{j}}=\frac{s_{j}}{\xi_{j}}=\frac{\left[D_{\mu}^{\prime} \eta_{j}^{2}+\left\{\rho_{2} c^{2}-\left(D_{\lambda}^{\prime}+2 D_{\mu}^{\prime}\right)\right\}\right]}{\left\{i\left(D_{\lambda}^{\prime}+D_{\mu}^{\prime}\right) \eta_{j}-\frac{i \rho_{2} g}{k}\right\}} \equiv m_{j} .
$$




\section{Boundary conditions and dispersion relation}

(i) At the interface, $z=0$, the continuity of the displacement along the $x$ direction requires that $u_{1}=u_{2}$ and $w_{1}=w_{2}$, where $u_{1}$ and $w_{1}$ are the displacement component in the layer along the $x$ and $z$ directions, respectively.

(ii) At the interface, $z=0$, the continuity of the stress requires that $\left(\tau_{x z}\right)_{\text {medium } 1}=\left(\tau_{x z}\right)_{\text {medium } 2}$ and $\left(\tau_{z z}\right)_{\text {medium } 1}=\left(\tau_{z z}\right)_{\text {medium } 2}$, where $\tau_{x z}$ and $\tau_{z z}$ are the relevant stress components.

(iii) At the upper boundary plane (free surface), that is, at $z=-h$, the stresses vanish, that is, $\left(\tau_{x z}\right)_{\text {medium } 1}=0$ and $\left(\tau_{z z}\right)_{\text {medium } 1}=0$.

$$
\begin{gathered}
\phi_{1}+\phi_{2}+\phi_{3}+\phi_{4}-\xi_{1}-\xi_{2}=0, \\
p_{1} \phi_{1}+p_{2} \phi_{2}+p_{3} \phi_{3}+p_{4} \phi_{4}-m_{1} \xi_{1}-m_{2} \xi_{2}=0,
\end{gathered}
$$$$
T_{1} \phi_{1}+T_{2} \phi_{2}+T_{3} \phi_{3}+T_{4} \phi_{4}-D_{\mu}^{\prime}\left(i m_{1}-\eta_{1}\right) \xi_{1}-D_{\mu}^{\prime}\left(i m_{2}-\eta_{2}\right) \xi_{2}=0,
$$$$
T_{5} \phi_{1}+T_{6} \phi_{2}+T_{7} \phi_{3}+T_{8} \phi_{4}-\left\{i D_{\lambda}^{\prime}-\left(D_{\lambda}^{\prime}+2 D_{\mu}^{\prime}\right)_{3} \eta_{1} m_{1}\right\} \xi_{1}
$$$$
-\left\{i D_{\lambda}^{\prime}-\left(D_{\lambda}^{\prime}+2 D_{\mu}^{\prime}\right)_{3} \eta_{2} m_{2}\right\} \xi_{2}=0 \text {, }
$$$$
T_{1} \phi_{1} e^{k \delta_{1} h}+T_{2} \phi_{2} e^{k \delta_{2} h}+T_{3} \phi_{3} e^{k \delta_{3} h}+T_{4} \phi_{4} e^{k \delta_{4} h}=0,
$$$$
T_{5} \phi_{1} e^{k \delta_{1} h}+T_{6} \phi_{2} e^{k \delta_{2} h}+T_{7} \phi_{3} e^{k \delta_{3} h}+T_{8} \phi_{4} e^{k \delta_{4} h}=0 \text {. }
$$

Eliminating $\phi_{1}, \phi_{2}, \phi_{3}, \phi_{4}, \xi_{1}$, and $\xi_{2}$ from Equations (29) to (34), we have

$\left|\begin{array}{cccccc}1 & 1 & 1 & 1 & -1 & -1 \\ p_{1} & p_{2} & p_{3} & p_{4} & -m_{1} & -m_{2} \\ T_{1} & T_{2} & T_{3} & T_{4} & -D_{\mu}^{\prime}\left(i m_{1}-\eta_{1}\right) & -D_{\mu}^{\prime}\left(i m_{2}-\eta_{2}\right) \\ T_{5} & T_{6} & T_{7} & T_{8} & -\left\{i D_{\lambda}^{\prime}-\left(D_{\lambda}^{\prime}+2 D_{\mu}^{\prime}\right)_{3} \eta_{1} m_{1}\right\} & -\left\{i D_{\lambda}^{\prime}-\left(D_{\lambda}^{\prime}+2 D_{\mu}^{\prime}\right)_{3} \eta_{2} m_{2}\right\} \\ T_{1} e^{k \delta_{1} h} & T_{2} e^{k \delta_{2} h} & T_{3} e^{k \delta_{3} h} & T_{4} e^{k \delta_{4} h} & 0 & 0 \\ T_{5} e^{k \delta_{1} h} & T_{6} e^{k \delta_{2} h} & T_{7} e^{k \delta_{3} h} & T_{8} e^{k \delta_{4} h} & 0 & 0\end{array}\right|=0$.

(iv) The displacement is bounded, that is, $\lim _{z \rightarrow \infty} u_{2}=0$ and $\lim _{z \rightarrow \infty} w_{2}=0$.

Now, using the boundary condition (iv), the solution of Equations (19) and (20) can be written as

$$
\begin{gathered}
u_{2}(x, z, t)=\left(\xi_{1} e^{-k \eta_{1} z}+\xi_{2} e^{-k \eta_{2} z}\right) e^{i k(x-c t)}, \\
w_{2}(x, z, t)=\left(m_{1} \xi_{1} e^{-k \eta_{1} z}+m_{2} \xi_{2} e^{-k \eta_{2} z}\right) e^{i k(x-c t)},
\end{gathered}
$$

Using the boundary conditions (i) to (iii), we have, respectively,

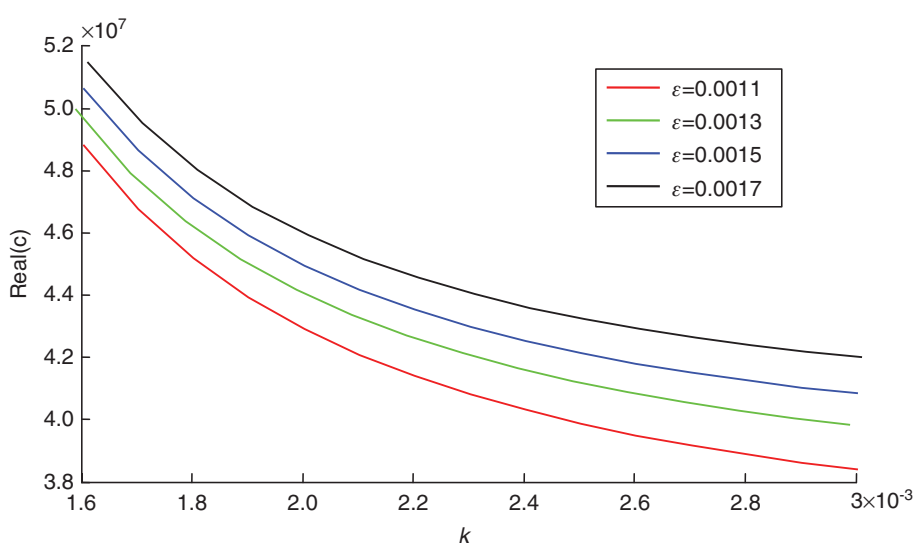

Figure 2 Variation of the phase velocity Real(c) against the wave number $k$ for a fixed depth $h=15 \mathrm{~km}$.
Equation (35) is the dispersion equation for the Rayleigh waves in an inhomogeneous anisotropic layer lying over an isotropic viscoelastic half-space of the higher order.

\section{Numerical results and discussion}

In order to show the effect of nonhomogeneity and phase velocity dependence on the wave number, we have taken the data from Vashishth and Sharma [12]. 
$a_{11}=17.77 \mathrm{Gpa}, a_{12}=3.78 \mathrm{Gpa}, a_{13}=3.76 \mathrm{Gpa}, a_{14}=0.24 \mathrm{Gpa}, a_{15}=-0.28 \mathrm{Gpa}$,

$a_{16}=0.03 \mathrm{Gpa}, a_{22}=19.45 \mathrm{Gpa}, a_{23}=4.13 \mathrm{Gpa}, a_{24}=-0.41 \mathrm{Gpa}, a_{25}=0.07 \mathrm{Gpa}$,

$a_{26}=1.13 \mathrm{Gpa}, a_{33}=21.79 \mathrm{Gpa}, a_{34}=-0.12 \mathrm{Gpa}, a_{35}=-0.01 \mathrm{Gpa}, a_{36}=0.38 \mathrm{Gpa}$,

$a_{44}=8.30 \mathrm{Gpa}, a_{45}=0.66 \mathrm{Gpa}, a_{46}=0.06 \mathrm{Gpa}, a_{55}=7.62 \mathrm{Gpa}, a_{56}=0.52 \mathrm{Gpa}, a_{66}=7.77 \mathrm{Gpa}$,

$\rho_{0}=2216 \mathrm{~kg} / \mathrm{m}^{3}$.

For a viscoelastic half-space of the higher order, the viscoelastic coefficients up to the third order are taken as follows:

$$
\begin{aligned}
& \lambda_{0}=1.25 \mathrm{Gpa}, \lambda_{1}=7.66 \mathrm{Gpa}, \lambda_{2}=2.5 \mathrm{Gpa}, \lambda_{3}=1.7 \mathrm{Gpa}, \\
& \mu_{0}=7.15 \mathrm{Gpa}, \mu_{1}=0.82 \mathrm{Gpa}, \mu_{2}=0.52 \mathrm{Gpa}, \mu_{3}=0.2 \mathrm{Gpa}, \\
& \rho_{2}=3380 \mathrm{~kg} / \mathrm{m}^{3} .
\end{aligned}
$$

The graphs are plotted separately for the real and imaginary parts of the phase velocity against the wave number with a MATLAB software. In Figure 2, the relevant graph is plotted for the real part of phase velocity Real(c) against the wave number $(k)$ for different values of the inhomogeneity parameter $\varepsilon(0.0011,0.0013,0.0015$, and 0.0017$)$ at a fixed depth $h=15 \mathrm{~km}$. The curves show that the phase velocity decreases for the increasing wave number. However, as we increase the inhomogeneity parameter $\varepsilon$, the magnitude of the phase velocity increases for all $k$ 's. The behavior of the curves is the same, but the curves are getting closer and closer with the increasing values of the wave number. It is clearly seen that the effect of inhomogeneity on the phase velocity is more significant for the small values of the wave number, and as the wave number increases, the effect of inhomogeneity decreases. In Figure 3, the relevant graph is plotted for the imaginary part of the phase velocity against the wave number $k$ for different values of the inhomogeneity parameter $\varepsilon(0.0011,0.0013,0.0015$, and 0.0017$)$ and the fixed depth $h=15 \mathrm{~km}$. The figure shows that the phase velocity decreases for increasing wave numbers, but as we increase $\varepsilon$, the phase velocity increases for all $k$ 's as before. In Figure 4, the relevant graph is plotted for the real part of the phase velocity against the wave number $k$ for $\varepsilon=0.0015$ and different depths $h=(0,10,20$, and 40$) \mathrm{km}$. It can be seen from the graph that the phase velocity decreases for the increasing wave number. As we increase the thickness of the layer, the phase velocity increases for some wave number $k$ 's, but all curves for different thicknesses coincide at $k=2.28 \times 10^{-3}$, and after that, the behavior of the phase velocity changes. It is shown that the thickness of the layer has significant effects on the phase velocity. In Figure 5, the relevant graph is plotted for the imaginary part of the phase velocity against the wave number $k$ for $\varepsilon=0.0015$ and the different depths $h=(0,10,20$, and 40) $\mathrm{km}$. The figure suggests that, as we increase the thickness of the layer, the phase velocity increases for all $k$ 's, but the behavior of the curve remains the same.

\section{Conclusions}

The Rayleigh wave propagation in an inhomogeneous anisotropic layer lying over an isotropic viscoelastic solid medium of the nth order under gravity has been

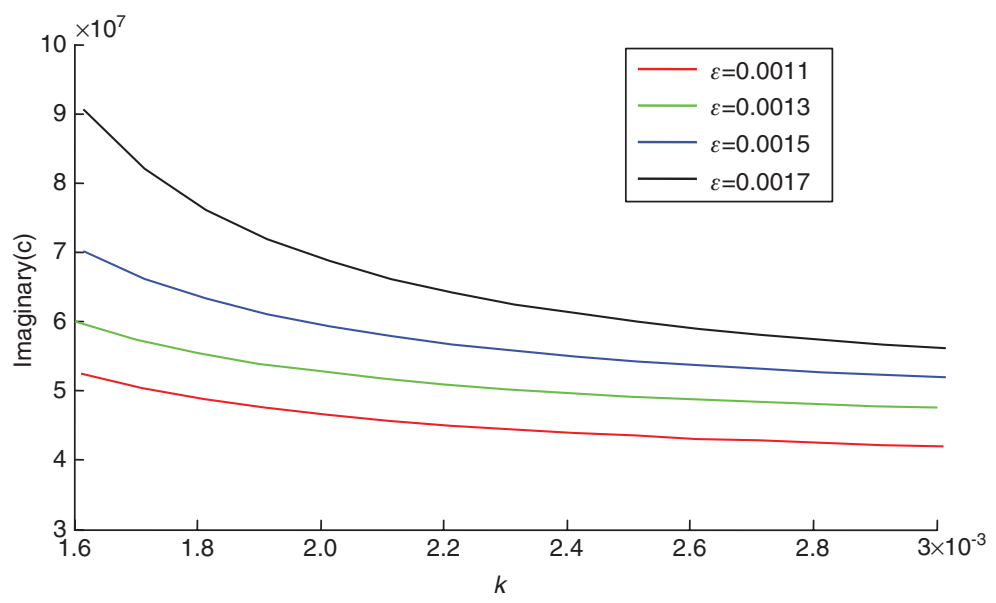

Figure 3 Variation of the phase velocity Imaginary(c) against the wave number $k$ for a fixed depth $h=15 \mathrm{~km}$. 


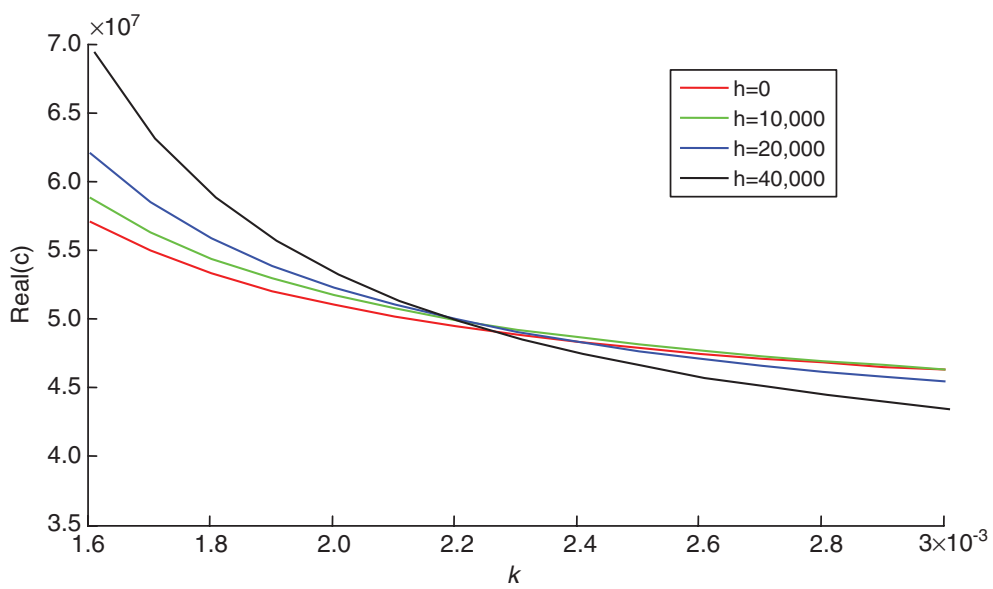

Figure 4 Variation of the phase velocity Real(c) against the wave number $k$ for a fixed inhomogeneity parameter $\varepsilon=0.0015$.

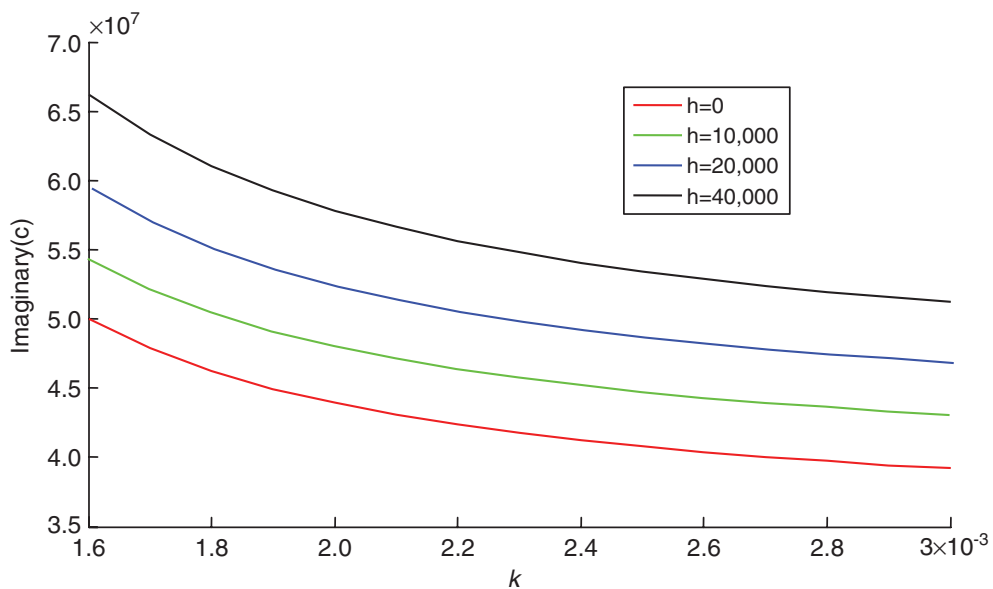

Figure 5 Variation of the phase velocity Imaginary(c) against the wave number $k$ for a fixed inhomogeneity parameter $\varepsilon=0.0015$.

investigated. The dispersion analysis shows that the phase velocity of the Rayleigh waves is influenced by the inhomogeneity, gravity, and strain rate parameters. The numerical results are discussed by plotting the corresponding graphs between the phase velocity and the wave number. It can be concluded from the graphs that the inhomogeneity parameter and thickness of the layer have a considerable effect on the phase velocity. The gravity parameter has no significant effect on the phase velocity.

\section{Appendix}

$$
\begin{array}{r}
\alpha_{0}=a_{33} a_{35}-a_{35}{ }^{2}, \alpha_{1}=-2\left\{i\left(a_{15} a_{33}-a_{13} a_{35}\right)+\frac{\varepsilon\left(a_{33} a_{55}-a_{35}{ }^{2}\right)}{k}\right\} \\
\alpha_{2}=\left(a_{33}+a_{55}\right) \rho_{0} c^{2}-4 a_{15} a_{35}{ }^{-} a_{11} a_{33}+a_{13}{ }^{2}+2 a_{13} a_{15}+2 a_{15} a_{35} \\
+\frac{3 i \varepsilon\left(a_{15} a_{33}-a_{13} a_{35}\right)}{k}+\frac{\varepsilon^{2}\left(a_{33} a_{55}-a_{35}{ }^{2}\right)}{k^{2}}
\end{array}
$$

$$
\alpha_{3}=-\left\{\begin{array}{r}
\left(2 i a_{15}+2 i a_{35}+\frac{\varepsilon a_{35}}{k}+\frac{\varepsilon a_{33}}{k}\right) \rho_{0} c^{2}-2 i a_{11} a_{35}+2 i a_{13} a_{15}+\frac{2 \varepsilon a_{13} a_{55}}{k}-\frac{2 \varepsilon a_{15} a_{35}}{k} \\
-\frac{\varepsilon a_{11} a_{33}}{k}+\frac{\varepsilon a_{13}{ }^{2}}{k}-\frac{i \varepsilon^{2} a_{13} a_{35}}{k}-\frac{i \varepsilon^{2} a_{35} a_{55}}{k}+\frac{i \varepsilon^{2} a_{35} a_{55}}{k^{2}}+\frac{i \varepsilon^{2} a_{33} a_{15}}{k^{2}}
\end{array}\right\}
$$




$$
\begin{aligned}
& \alpha_{4}=\rho_{0}{ }^{2} c^{4}-\left(a_{55}+a_{11}+\frac{i \varepsilon a_{35}}{k}\right) \rho_{0} c^{2}+a_{11} a_{55}-a_{15}{ }^{2}-\frac{i \varepsilon a_{11} a_{35}}{k} \\
& T_{7}=i a_{13}-p_{3} \delta_{3} a_{33}+\left(i p_{3}-\delta_{3}\right) a_{35}, K_{8}=i a_{13}-p_{4} \delta_{4} a_{33}+\left(i p_{4}-\delta_{4}\right) a_{35} . \\
& +\frac{i \varepsilon a_{13} a_{15}}{k}-\frac{i \rho_{0} g a_{15}}{k}+\frac{i \varepsilon a_{15} a_{55}}{\rho}+\frac{\varepsilon^{2} a_{13} a_{55}}{k^{2}}-\frac{\varepsilon \rho_{0} g a_{55}}{k^{2}} \\
& \beta_{0}=D_{\mu}^{\prime}\left(D_{\lambda}^{\prime}+2 D_{\mu}^{\prime}\right) \\
& \beta_{1}=D_{\mu}^{\prime}\left(\rho_{2} c^{2}-D_{\mu}^{\prime}\right)+\left(D_{\lambda}^{\prime}+2 D_{\mu}^{\prime}\right)\left\{\rho_{2} c^{2}-\left(D_{\lambda}^{\prime}+2 D_{\mu}^{\prime}\right)\right\}+\left(D_{\lambda}^{\prime}+D_{\mu}^{\prime}\right)^{2} \\
& \beta_{2}=\left(\rho_{2} c^{2}-D_{\mu}^{\prime}\right)\left\{\rho_{2} c^{2}-\left(D_{\lambda}^{\prime}+2 D_{\mu}^{\prime}\right)\right\}-\frac{\rho_{2}^{2} g^{2}}{k^{2}} \\
& T_{1}=i a_{15}-p_{1} \delta_{1} a_{35}+\left(i p_{1}-\delta_{1}\right) a_{55}, T_{2}=i a_{15}-p_{2} \delta_{2} a_{35}+\left(i p_{2}-\delta_{2}\right) a_{55} \\
& T_{3}=i a_{15}-p_{3} \delta_{3} a_{35}+\left(i p_{3}-\delta_{3}\right) a_{55}, T_{4}=i a_{15}-p_{4} \delta_{4} a_{35}+\left(i p_{4}-\delta_{4}\right) a_{55} \\
& T_{5}=i a_{13}-p_{1} \delta_{1} a_{33}+\left(i p_{1}-\delta_{1}\right) a_{35}, T_{6}=i a_{13}-p_{2} \delta_{2} a_{33}+\left(i p_{2}-\delta_{2}\right) a_{35}
\end{aligned}
$$

\section{References}

